\title{
Long-Wave Coupled Marangoni - Rayleigh Instability in a Binary Liquid Layer in the Presence of the Soret Effect
}

\author{
A. Podolny ${ }^{a}$, A.A. Nepomnyashchy ${ }^{a, b}$ and A. Oron ${ }^{c 1}$ \\ ${ }^{a}$ Department of Mathematics, Technion - Israel Institute of Technology, Haifa, 32000, Israel. \\ ${ }^{b}$ Minerva Center for Nonlinear Physics of Complex System, \\ Technion - Israel. Institute of Technology, Haifa, 32000, Israel \\ ${ }^{c}$ Department of Mechanical Engineering, Technion- Israel Institute of Technology, Haifa, Israel.
}

\begin{abstract}
We have explored the combined long-wave Marangoni and Rayleigh instability of the quiescent state of a binary- liquid layer heated from below or from above in the presence of the Soret effect. We found that in the case of small Biot numbers there are two longwave regions of interest $k \sim B i^{1 / 2}$ and $k \sim B i^{1 / 4}$. The dependence of both monotonic and oscillatory thresholds of instability in these regions on both the Soret and dynamic Bond numbers has been investigated. The complete linear stability analysis reveals the diversity of instability types in the long-wave region, and a need in the development of the nonlinear theory of the discovered phenomena becomes obvious.
\end{abstract}

Key words: binary liquid, Marangoni instability, buoyancy, Soret effect AMS subject classification: 76E06, 76E17

\section{Introduction}

Various transport processes encountered in technology and nature are due to or affected by simultaneous action of temperature and solute concentration gradients. Different configurations of those gradients were discussed [16] in the context of buoyancy-driven convection. Similar settings can be also considered in regards with the surface-tension-driven convection in the no-gravity environment. Relevant examples are different techniques of materials

\footnotetext{
${ }^{1}$ Corresponding author. Email: asspekto@tx.technion.ac.il
} 
processing, e.g. crystal growth, from binary or multicomponent liquid mixtures. Many of them, especially those employing the floating zone and temperature-gradient methods, involve large temperature and possibly concentration gradients imposed in various directions relatively to the melt [16].

The buoyancy-driven (Rayleigh) convection in binary mixtures has been a subject of an extensive investigation, in both theoretical and experimental aspects. It is now well-known that simultaneous presence of two or more components with different diffusivities in a liquid layer may lead to a variety of new phenomena. Specifically, if two or more components with different diffusivities are present in a fluid and their gradients make opposing contributions to the fluid density, this can create a possible source of instability. For instance, a layer subjected to a stabilizing solute concentration can exhibit an oscillatory instability when a destabilizing thermal gradient across it opposes the former [13]. Two main physical situations are possible here: (i) the temperature gradient and the concentration gradient have independent sources (double-diffusive convection); (ii) the temperature gradient is imposed, while the concentration gradient is generated spontaneously due to the Soret effect[8]. Extensive reviews $[25,26]$ encompass the work on double-diffusive phenomena and their applications in oceanography, chemistry, metallurgy, geology, geophysics, etc. A review on the convection in layers of a binary liquid with the Soret effect can be found in Ref.[6].

If the liquid layer has a free surface, the surface-tension- driven (Marangoni) convection, caused by the dependence of the surface tension on both the temperature and the solute concentration, can appear [5], [12]. In a majority of mixtures surface tension decreases with temperature and increases (decreases) with concentration of an inorganic (organic) solute. Therefore, if a layer of a binary mixture is subjected to both temperature and concentration gradients, nonuniformities of those at the free surface lead to the emergence of surface shear stresses that can under certain conditions destabilize the quiescent base state.

When a binary fluid layer with a nonzero Soret effect is subjected to a temperature gradient across the layer, a concomitant solute concentration gradient will be induced. The ratio of these two gradients is proportional to the separation ratio $\chi$, a property of the liquid. Similarly, the ratio of the thermal and solutal Marangoni numbers, which are nondimensional increments in surface tension due to changes in temperature and concentration, is also proportional to the separation ratio. As a consequence, the stability of a given binary fluid layer with a free surface under zero gravity depends only on the temperature difference $\Delta T$, imposed across the layer or, on the thermal Marangoni number $M$. When the gravity is nonzero but of a small magnitude, such that the buoyancy effects are not dominant, the stability characteristics of the layer are functions of two parameters, $M$ and $R$, the thermal Rayleigh number.

As mentioned above, the concentration gradient across the layer can be either independent of the temperature gradient or generated by the Soret effect. The linear stability analysis of the quiescent equilibrium in a layer with a free surface under the action of independent temperature and concentration gradients across the layer was carried out in [1]-[4],[11],[21],[23],[24]. The case where the solute concentration is produced by the Soret effect, was considered in [1]-[3],[21]-[24]. 
The problem of the onset of surface tension driven instability in a horizontal layer of binary liquid mixture subjected to a vertical temperature gradient is examined in the framework of linear stability theory [23]. The solute is assumed to be soluble and the Soret effect is taken into account. When a binary fluid layer with a nonzero Soret effect is subjected to a temperature gradient across the layer, a concomitant solute concentration gradient will be induced. The ratio of these two gradients is proportional to the separation ratio, a material property of the binary liquid.

This theory was extended in [24] to include the possibility of oscillatory instability. Linear stability theory was applied to derive a time-dependent eigenvalue relationship which was then computed numerically. It was shown there that depending on the values of various dimensionless parameters, such as Marangoni, Biot, Prandtl and Schmidt numbers, instability can set in either as stationary convection or as overstability.

Stability criteria for experiments in standard and reduced-gravity conditions were derived in the case when the Soret effect is acting in a horizontal binary liquid layer heated from below and open for the ambient gas [1], [2]. Sufficient conditions for the emergence of either monotonic or oscillatory Rayleigh-Benard convection were discussed in the case of a horizontal, two-component liquid layer heated or cooled from below with a non-deformable gas-liquid interface. The effect of the Soret separation (thermal diffusion), and its coupling to Marangoni tractions induced by temperature and/or composition inhomogeneities at the open interface were also considered. Specific predictions of relevance to experiments in microgravity conditions were given.

The influence of the nonlinear Soret effect on the stationary Marangoni-Benard stability conditions in a binary mixture layer with a flat interface was studied in Ref. [22]. Linear stability analysis showed that long-wave instabilities are possible depending on the value of the Soret number $\chi$. Weakly nonlinear evolution equations for both monotonic and oscillatory Marangoni instabilities in a binary liquid in the presence of the Soret effect were also derived [15]. Pattern formation in the framework of these equations was studied [15], [19].

It is the purpose of the present work to study the important case of the convection in a binary liquid layer with poorly conducting boundaries, where the quiescent state is unstable with respect to long-wave disturbances. This paper presents an extension of the linear stability analysis carried out in Ref.[15] to the case of the coupled Rayleigh-Marangoni convection in a binary solution. Here, in the limit when the heat flux across the layer is fixed, uniform variations of the temperature and solutal concentration are neither damped nor amplified. When large-scale horizontal modulation of both the temperature and concentration fields is imposed, flows are generated by both surface-tension and density gradients which can lead to a long-wave instability. In the present work we carry out the linear stability analysis of the system and find long-wave both monotonic and oscillatory modes of instability in various parameter domains. 


\section{Statement of the problem and governing equations}

\subsection{Mathematical model}

We consider a two-dimensional horizontal layer of an incompressible binary liquid of an infinite extent in the longitudinal direction $x$ lying on a rigid plane and exposed to the ambient gas phase at its nondeformable free surface. The layer of thickness $h_{0}$ is subjected to a temperature gradient $-a$ across the film. It is assumed that the film is not too thin, so that the effect of buoyancy cannot be neglected as compared to the impact of the Marangoni effect. The liquid density $\rho$ is assumed to depend on both the temperature $\vartheta$ and solute concentration $c$,

$$
\rho=\rho_{0}\left[1-\widetilde{\beta}\left(\vartheta-\vartheta_{0}\right)+\widetilde{\gamma}\left(c-c_{0}\right)\right],
$$

where $\rho_{0}, \vartheta_{0}, c_{0}$ are, respectively, reference values of density, temperature and solute concentration, $\widetilde{\beta}=-\frac{1}{\rho_{0}}\left(\frac{\partial \rho}{\partial \vartheta}\right)_{p}, \quad \widetilde{\gamma}=\frac{1}{\rho_{0}}\left(\frac{\partial \rho}{\partial c}\right)_{p}$. Thus, the effect of buoyancy is included in the analysis. Surface tension $\sigma$ is also assumed to be dependent upon both temperature $\vartheta$ and solute concentration $c$,

$$
\begin{gathered}
\sigma(\vartheta, c)=\sigma_{0}-\sigma_{t}\left(\vartheta-\vartheta_{0}\right)+\sigma_{c}\left(c-c_{0}\right) \\
\sigma_{t}=-\partial \sigma / \partial \vartheta, \quad \sigma_{c}=\partial \sigma / \partial c,
\end{gathered}
$$

where $\sigma_{0}=\sigma\left(\vartheta_{0}, c_{0}\right)$, and therefore, both thermo- and solutocapillary effects are taken into consideration.

As a generalization of the standard model of heat and mass transfer given by the Fourier's and Fick's laws, where the heat and mass fluxes are taken proportional to the temperature and concentration gradients, respectively, it is appropriate to consider both fluxes to be each linear combinations of both of these gradients [8]. This extended model incorporates the Soret and Dufour effects. However, the Dufour effect is exceedingly weak in liquids and can be safely neglected [9], while the Soret effect can be significant and will be taken into account hereafter. Therefore, in what follows the heat $J_{h}$ and mass $J_{m}$ fluxes are given by

$$
J_{h}=-\lambda \vec{\nabla} \vartheta, J_{m}=-\rho D(\vec{\nabla} c+\alpha \vec{\nabla} \vartheta)
$$

respectively, where $\lambda, D, \alpha$ represent thermal conductivity, mass diffusivity and the Soret coefficient of the binary mixture, respectively.

A set of governing equations consists of the two-dimensional Navier-Stokes equations for an incompressible liquid in the Boussinesq approximation, as well as heat and mass transfer equations in the presence of the Soret effect

$$
\begin{gathered}
\nabla \cdot \vec{v}=0 \\
\vec{v}_{t}+(\vec{v} \cdot \nabla) \vec{v}=-\rho_{0}^{-1} \nabla p+\nu \nabla^{2} \vec{v}+g \widetilde{\beta}(\vartheta-\bar{\vartheta}) \vec{e}_{z}-g \widetilde{\gamma}(c-\bar{c}) \vec{e}_{z} .
\end{gathered}
$$




$$
\begin{gathered}
\vartheta_{t}+\vec{v} \cdot \nabla \vartheta=\kappa \nabla^{2} \vartheta, \\
c_{t}+\vec{v} \cdot \nabla c=D \nabla^{2} c+\alpha D \nabla^{2} \vartheta,
\end{gathered}
$$

Here $\vec{v}=(u, w), p$ are fields of the fluid velocity and pressure, respectively, $\nu, \kappa$ are, respectively, kinematic viscosity, and thermal diffusivity of the binary mixture, $\vec{\nabla} \equiv\left(\partial_{x}, \partial_{z}\right), t$ is time, and $\bar{\vartheta}, \bar{c}$ are constant average values of the temperature and concentration, respectively. The $x$-axis is directed along the solid substrate, while the $z$-axis is normal to the latter.

The boundary conditions at the bottom rigid surface $z=0$ reflect the no-slip, nopenetration conditions for the velocities, a specified heat flux and mass impermeability, respectively,

$$
z=0: \quad \vec{v}=0, \quad \vartheta_{z}=-a, \quad c_{z}=\alpha a,
$$

At the free non-deformable liquid-gas interface, the boundary conditions are, respectively, the kinematic boundary condition, heat transfer governed by the Newton's law of cooling and mass impermeability, respectively,

$$
\begin{gathered}
z=h_{0}: \quad \vec{v} \cdot \vec{e}_{z}=0, \quad k_{t h} \nabla \vartheta \cdot \vec{n}+q\left(\vartheta-\vartheta_{\infty}\right)=0, \\
k_{t h} \nabla c \cdot \vec{n}-\alpha \cdot q\left(\vartheta-\vartheta_{\infty}\right)=0
\end{gathered}
$$

where $k_{t h}$ is the thermal conductivity of the mixture, $q$ is the rate of heat transfer by convection at the free surface, $\vec{n}$ is the normal vector in the $z$ direction, and $\vartheta_{\infty}$ is the sustained temperature of the ambient gas phase. Also, the balance of tangential stresses at the free surface is given by

$$
\mu \partial_{z} u=-\sigma_{t} \frac{\partial \vartheta}{\partial x}+\sigma_{c} \frac{\partial c}{\partial x}
$$

where $\mu$ is dynamic viscosity of the fluid. We define the dimensionless variables of the problem as

$$
\begin{gathered}
t \rightarrow \frac{h_{0}^{2}}{\nu} t,(x, z) \rightarrow h_{0}(x, z),(u, w) \rightarrow \frac{\kappa}{h_{0}}(u, w), \\
\vartheta \rightarrow \vartheta_{\infty}+a h_{0} T, c \rightarrow \frac{\sigma_{t}}{\sigma_{c}} a h_{0} C, p \rightarrow \frac{\mu \kappa}{h_{0}^{2}} p .
\end{gathered}
$$

This yields the dimensionless form of governing equations:

$$
\begin{gathered}
\nabla \cdot \vec{v}=0 \\
\vec{v}_{t}+P^{-1}(\vec{v} \cdot \nabla) \vec{v}=-\nabla p+\nabla^{2} \vec{v}+R_{t h}(T-\bar{T}) \cdot \vec{e}_{z}-R_{c}(C-\bar{C}) \cdot \vec{e}_{z}, \\
P T_{t}+\vec{v} \cdot \nabla T=\nabla^{2} T \\
S C_{t}+L^{-1} \vec{v} \cdot \nabla C=\nabla^{2} C+\chi \nabla^{2} T,
\end{gathered}
$$

and the boundary conditions are rewritten as:

$$
z=0: \quad \vec{v}=0, \quad T_{z}=-1, \quad C_{z}=\chi
$$




$$
\begin{gathered}
z=1: \quad \vec{v} \cdot \vec{e}_{z}=0, \quad T_{z}+B i T=0, \quad C_{z}-\chi B i T=0, \\
\partial_{z} u+M_{t h} \partial_{x}(T-C)=0 .
\end{gathered}
$$

The dimensionless parameters of the problem that appear in Eqs.(2.12)- (2.14) are $P=\frac{\nu}{\kappa}$ - Prandtl number, $S=\frac{\nu}{D}$ - Schmidt number, $\chi=\frac{\alpha \sigma_{c}}{\sigma_{t}}$ - Soret number, $B i=\frac{q h_{0}}{k_{t h}}$ - Biot number, $M_{t h}=\frac{\sigma_{t} a h_{0}^{2}}{\mu \kappa}$ - thermal Marangoni number, $R_{t h}=\frac{g \widetilde{\beta} a h_{0}^{4}}{\mu \kappa}$ - thermal Rayleigh number, $R_{c}=\frac{g \widetilde{\gamma} \sigma_{t} a h_{0}^{4}}{\sigma_{c} \mu \kappa}$ - solutal Rayleigh number, and $\Sigma=\frac{\sigma h_{0}}{\mu \kappa}$ - inverse capillary number. In the following analysis, we also use $M$ as a substitute for $M_{t h}$.

In what follows, we will also make use of the Lewis number $L=\frac{P}{S}$. It should be emphasized that in virtually all physical settings $S \gg P$ and therefore, the relevant range for the Lewis number is $L \ll 1$.

\section{$2.2 \quad$ Linearized problem}

The base state whose stability is investigated is given by

$$
\begin{gathered}
\vec{v}_{0}=0, \quad T_{0}=-z+\frac{1+B}{B}, \quad C_{0}=\chi z+C_{\omega}, \\
p_{0}=-\frac{R_{t h}}{2} z^{2}(1+\chi \varphi)+R_{t h}\left\{\frac{1+B i}{B i}-\bar{T}+\varphi\left(\bar{C}-C_{\omega}\right)\right\} \cdot z+\text { const }
\end{gathered}
$$

where

$$
\varphi=\frac{R_{c}}{R_{t h}}=\frac{\widetilde{\gamma} \sigma_{t}}{\widetilde{\beta} \sigma_{c}}
$$

is the separation ratio, $C_{\omega}$ is the dimensionless solute concentration at $z=0$ and const is an arbitrary constant.

Linearization of the governing equations (2.12) around the base state (2.15) results in the following system of equations for perturbation functions which are denoted by the same symbols as the corresponding fields themselves

$$
\begin{gathered}
\nabla \cdot \vec{v}=0, \\
\vec{v}_{t}=-\nabla p+\nabla^{2} \vec{v}+R_{t h}(T-\varphi C) \vec{e}_{z}, \\
P T_{t}-w=\nabla^{2} T, \\
P L^{-1} C_{t}+L^{-1} \chi w=\nabla^{2} C+\chi \nabla^{2} T .
\end{gathered}
$$


The boundary conditions (2.13) and (2.14) are rewritten as at $z=0$ :

$$
\vec{v}=0, \quad T_{z}=C_{z}=0
$$

at $z=1$ :

$$
\begin{gathered}
w=0, \quad T_{z}+B i T=0, \quad C_{z}-\chi B i T=0, \\
u_{z}=M_{t h}\left(C_{x}-T_{x}\right),
\end{gathered}
$$

The components of the velocity field are hereafter expressed in terms of the stream function $\psi$ as

$$
u=\psi_{z}, \quad w=-\psi_{x} .
$$

We consider the normal modes in the form

$$
(\psi, p, T, C, \psi)=(\widetilde{\psi}(z), \widetilde{p}(z), \widetilde{T}(z), \widetilde{C}(z)) e^{i k x+\omega t},
$$

where $k$ and $\omega$ are wave number and growth rate of the disturbances, respectively.

Substituting Eq. (2.19) into Eqs. (2.16), (2.17) and (2.18) yields

$$
\begin{gathered}
\omega\left(\widetilde{\psi}^{\prime \prime}-k^{2} \widetilde{\psi}\right)=\widetilde{\psi}^{\prime \prime \prime \prime}-2 k^{2} \widetilde{\psi}^{\prime \prime}+k^{4} \widetilde{\psi}-i k R_{t h}(\widetilde{T}-\varphi \widetilde{C}), \\
\omega P \widetilde{T}+i k \widetilde{\psi}=-k^{2} \widetilde{T}+\widetilde{T}^{\prime \prime} \\
\omega P L^{-1} \widetilde{C}-i k L^{-1} \chi \widetilde{\psi}=-k^{2} \widetilde{C}+\widetilde{C}^{\prime \prime}+\chi\left(-k^{2} \widetilde{T}+\widetilde{T}^{\prime \prime}\right) .
\end{gathered}
$$

The boundary conditions are

at $z=0$ :

$$
\widetilde{\psi}=\widetilde{\psi}^{\prime}=0, \quad \widetilde{T}^{\prime}=\widetilde{C}^{\prime}=0
$$

at $z=1$ :

$$
\begin{array}{cl}
\widetilde{\psi}=\widetilde{\psi}^{\prime}=0, & \widetilde{T}_{z}+B i \widetilde{T}=0, \quad \widetilde{C}_{z}-\chi B i \widetilde{T}=0, \\
\widetilde{\psi}^{\prime \prime}=i k M_{t h}(\widetilde{C}-\widetilde{T}) .
\end{array}
$$

In this paper we study the case of the long-wave instability of the system with poorly conducting boundaries. When both the wave number $k$ and the Biot number $B i$ tend to zero, the behavior of the critical Marangoni number $M_{t h}$ depends on the relationship between $k$ and $B i$. This was also the case in the pure Marangoni instability of binary liquids [15], where the case of $k=O\left(B i^{1 / 4}\right)$ was investigated. This limit was also studied for pure liquids [17], [14], [20], [7]. In addition to the standard long-wave regime $k=O\left(B i^{1 / 4}\right)$, a novel long-wave asymptotic domain $k=O\left(B i^{1 / 2}\right)$, special for binary fluids was found and investigated [18]. Therefore, in order to obtain the complete neutral curve, we consider both of these limits. In the former, we find that $T_{0} \sim C_{0}$, i.e. the instability is caused by both thermocapillary and solutocapillary effects. In the latter, we show that $T_{0} \sim \frac{\left(k^{2} / B i\right)}{\left(k^{2} / B i\right)+1} C_{0}$, i.e. the contribution of the thermocapillary effect is determined by the value of $k^{2} / B i$. 
In each of these cases, we introduce the scaling for the growth rate and stream function

$$
\omega=\varepsilon^{2}\left(\omega_{0}+\varepsilon^{2} \omega_{2}+\ldots\right), \widetilde{\psi}=\varepsilon \widetilde{\Psi},
$$

and the perturbation functions are expanded in the form

$$
(\widetilde{\Psi}, \widetilde{T}, \widetilde{C})=\left(\Psi_{0}, T_{0}, C_{0}\right)+\varepsilon^{2}\left(\Psi_{2}, T_{2}, C_{2}\right)+\ldots
$$

Here, $\varepsilon$ is a small parameter serving as a measure of supercriticality which will be defined later. The thermal Marangoni and Rayleigh numbers are expanded near the stability threshold as

$$
M_{t h}=M_{0}+\varepsilon^{2} M_{2}+\ldots, R_{t h}=R_{0}+\varepsilon^{2} R_{2}+\ldots
$$

\section{$3 \quad$ Linear stability analysis}

As noted above in contradistinction with the case of a pure fluid, where the consideration of the limit $k=O\left(B^{1 / 4}\right)$ was sufficient for finding the critical Marangoni number at $B i \ll 1$, the Marangoni convection in a binary fluid is characterized by two distinct asymptotic limits $k=O\left(B i^{1 / 4}\right)$ and $k=O\left(B i^{1 / 2}\right)[18]$. Linear stability analysis, carried out in [18] shows that in the region $k=O\left(B i^{1 / 4}\right)$, the monotonic neutral curve is given by $M(k)=M_{0}+\left(c_{1} k^{2}+\right.$ $\left.\varepsilon^{2} c_{2} k^{-2}\right)$, thus, for certain values of parameters $\left(M_{0}<0, c_{1}>0, c_{2}>0\right)$ it changes sign at $k_{c}^{2} \approx \varepsilon^{4} c_{2} /\left(-M_{0}\right) \ll 1$, and becomes negative in the range of $k=O(\varepsilon)$. This difficulty can be resolved by investigating in the novel long-wave domain $B i \sim k^{2}$.

\subsection{The case of $k=O\left(B i^{1 / 4}\right)$}

First, similar to the case of pure Marangoni instability in binary liquids[15] the scaling of the disturbance wavenumber and the Biot number is chosen as

$$
k=\varepsilon K, \quad B i=\varepsilon^{4} \beta,
$$

where $K$ is a rescaled wavenumber. This scaling along with Eqs.(2.23)-(2.25) is substituted into Eqs.(2.20)-(2.22) and a hierarchy of boundary-value problems emerges at each order.

At zero order of approximation we obtain

$$
\begin{gathered}
\Psi_{0}^{\prime \prime \prime \prime}-i K R_{0}\left(T_{0}-\varphi C_{0}\right)=0, \\
T_{0}^{\prime \prime}=0, \\
C_{0}^{\prime \prime}+\chi T_{0}^{\prime \prime}=0, \\
z=0: \quad \Psi_{0}=\Psi_{0}^{\prime}=0, T_{0}^{\prime}=C_{0}^{\prime}=0, \\
z=1: \quad \Psi_{0}=0, \quad T_{0}^{\prime}=C_{0}^{\prime}=0, \Psi_{0}^{\prime \prime}=i K M_{0}\left[C_{0}-T_{0}\right] .
\end{gathered}
$$


The solution of the problem (3.27) is given by

$$
\begin{gathered}
\Psi_{0}=\frac{i}{48} K(z-1) z^{2}\left(\left((2 z-3) R_{0}-12 M_{0}\right) a_{1}+a_{2}\left(12 M_{0}-(2 z-3) R_{0} \varphi\right)\right) \\
T_{0}=a_{1}, \quad C_{0}=a_{2}
\end{gathered}
$$

where $a_{1}$ and $a_{2}$ are constants.

At second order the equations and boundary conditions read

$$
\begin{gathered}
\Psi_{2}^{\prime \prime \prime \prime}-2 K^{2} \Psi_{0}^{\prime \prime}-i K R_{0}\left(T_{2}-\varphi C_{2}\right)-i K R_{2}\left(T_{0}-\varphi C_{0}\right)=\omega_{0} \Psi_{0}^{\prime \prime}, \\
T_{2}^{\prime \prime}-K^{2} T_{0}=i K \Psi_{0}+\omega_{0} P T_{0}, \\
C_{2}^{\prime \prime}-K^{2} C_{0}+\chi\left(T_{2}^{\prime \prime}-K^{2} T_{0}\right)=-i K \chi L^{-1} \Psi_{0}+\omega_{0} P L^{-1} C_{0}, \\
z=0: \quad \Psi_{2}=\Psi_{2}^{\prime}=0, \quad T_{2}^{\prime}=C_{2}^{\prime}=0, \\
z=1: \quad \Psi_{2}=0, \quad T_{2}^{\prime}=C_{2}^{\prime}=0, \\
\Psi_{2}^{\prime \prime}=i K M_{0}\left[C_{2}-T_{2}\right]+i K M_{2}\left[C_{0}-T_{0}\right] .
\end{gathered}
$$

The solvability conditions at second order obtained by integrating the heat and mass transfer equations over $0 \leq z \leq 1$, read

$$
\begin{gathered}
-K^{2}<T_{0}>=i K<\Psi_{0}>+\omega_{0} P<T_{0}> \\
-K^{2}<C_{0}>-\chi K^{2}<T_{0}>=-i K \chi L^{-1}<\Psi_{0}>+\omega_{0} P L^{-1}<C_{0}>
\end{gathered}
$$

where $<f>=\int_{0}^{1} f d z$.

Using Eqs.(3.28) we obtain a homogeneous set of linear equations

$$
\begin{gathered}
\mathbf{A}_{\mathbf{1}}\left(\begin{array}{l}
a_{1} \\
a_{2}
\end{array}\right)=\left(\begin{array}{l}
0 \\
0
\end{array}\right), \\
\mathbf{A}_{\mathbf{1}}=\left(\begin{array}{cl}
1-m_{0}-r_{0}+P \Lambda_{0} & m_{0}+r_{0} \varphi \\
\chi\left[\left(1+L^{-1}\right)\left(m_{0}+r_{0}\right)-P \Lambda_{0}\right] & 1+P L^{-1} \Lambda_{0}-\left(1+L^{-1}\right) \chi\left\{m_{0}+r_{0} \varphi\right\}
\end{array}\right),
\end{gathered}
$$

where $m_{0}=M_{0} / 48, r_{0}=R_{0} / 320$ and $\omega_{0}=\Lambda_{0} K^{2}$.

The dependence of the growth rate on the Marangoni number arising from the zero value of the determinant of the matrix $\mathbf{A}_{1}$, is determined by the quadratic equation

$$
\begin{gathered}
L-(L+\chi+L \chi) m_{0}+(-(1+L) \varphi \chi-L) r_{0}+ \\
P\left(1+L-(1+\chi) m_{0}-(\varphi \chi+1) r_{0}\right) \Lambda_{0}+P^{2} \Lambda_{0}^{2}=0 .
\end{gathered}
$$


The explicit expression for $\Lambda_{0}\left(m_{0}\right)$ reads

$$
\frac{\Lambda_{0}=\frac{1}{2 P}\left\{-1-L+m_{0}+\chi m_{0}+r_{0}+\chi r_{0} \varphi \pm\right.}{\left.\sqrt{\left(1+L-(1+\chi) m_{0}-r_{0}(1+\chi \varphi)\right)^{2}+4\left(-L+(L+\chi+L \chi) m_{0}+r_{0}(L+(1+L) \chi \varphi)\right)}\right\}} .
$$

Both of the cases of monotonic and oscillatory instabilities are studied based on Eq.(3.34).

\subsubsection{Monotonic instability}

We first consider the case of monotonic instability, thus we set $\omega_{0}=\omega_{2}=\ldots=0$.

Substituting $\Lambda_{0}=0$ into Eq.(3.33), we find that the monotonic instability threshold is determined by

$$
\frac{m_{0}}{\left[1+\chi+\chi L^{-1}\right]^{-1}}+\frac{r_{0}}{\left[\left(1+L^{-1}\right) \chi \varphi+1\right]^{-1}}=1
$$

Equation (3.35) reduces in the case of a pure liquid formally obtained at $\chi=0$ to the expression derived by Nield[14].

Introducing the dynamic Bond number as the ratio $B=\frac{R_{t h}}{M_{t h}}=\frac{g \widetilde{\beta} h_{0}^{2}}{\sigma_{t}}$, one can rewrite the expression for the monotonic instability threshold in the form

$$
m_{0}=\frac{1}{1+\widehat{B}+\left(1+L^{-1}\right) \tilde{\chi}}
$$

where $\widehat{B}=\frac{3}{20} B, \tilde{\chi}=\chi(1+\widehat{B} \varphi)$.

The critical Marangoni number is positive if $\tilde{\chi}>\tilde{\chi}_{1}$

$$
\widetilde{\chi}_{1}=-\frac{1+\widehat{B}}{1+L^{-1}}
$$

and negative if $\tilde{\chi}<\tilde{\chi}_{1}$.

Differentiating Eq.(3.33) with respect to $m_{0}$, we find that along the neutral curve

$$
\frac{d \Lambda_{0}}{d m_{0}}=\frac{[\tilde{\chi}+L(1+\widehat{B}+\tilde{\chi})]^{2}}{P[\tilde{\chi}+L(\chi+L(1+\widehat{B}+\tilde{\chi}))]} .
$$

Thus, $\frac{d \Lambda_{0}}{d m_{0}}$ changes its sign through infinity at the point

$$
\widetilde{\chi}_{2}=-\frac{1+\widehat{B}}{1+L^{-1}+L^{-2}} .
$$


To determine whether monotonic instability sets in when $|m|,\left(m \equiv M_{t h} / 48\right)$ becomes larger than $\left|m_{0}\right|$, we calculate the value $Q=m_{0} \frac{d \Lambda_{0}}{d m_{0}}$ along the neutral monotonic curve. If $Q$ is positive, i.e., $\frac{d \Lambda_{0}}{d m_{0}}$ and $m_{0}$ have the same sign, the value given by Eq.(3.36) indeed defines the monotonic instability threshold. Otherwise, it describes the stabilization boundary and this would suggest that an oscillatory neutral curve is located in the domain where $\mid \mathrm{m} \equiv$ $M_{t h} / 48|<| m_{0} \mid$. The variation of the rescaled growth rate on the Marangoni number in the vicinity of the critical point $m_{0}$ in various regions of $\widetilde{\chi}$ is presented in Figs. 1 and 2.

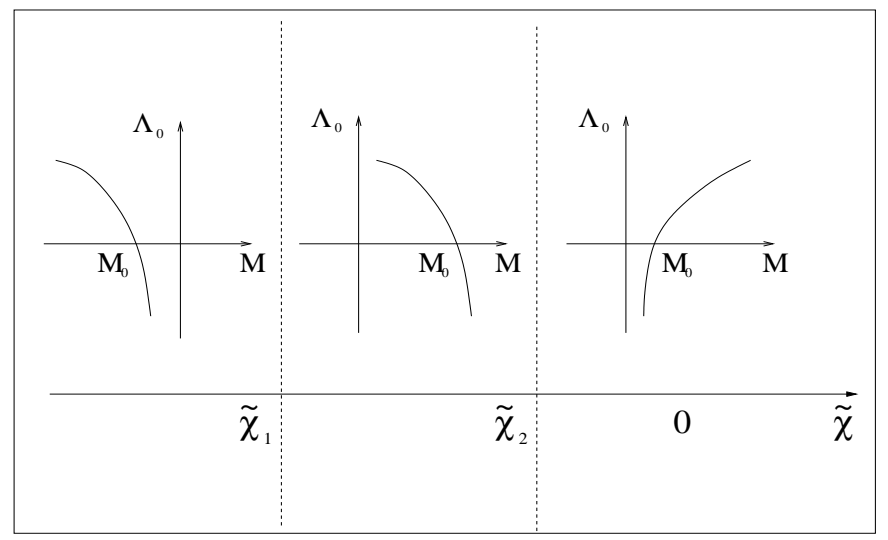

Figure 1: The dependence of the rescaled growth rate on the Marangoni number in the vicinity of the critical point $M_{0}=48 m_{0}$ in the different regions of $\tilde{\chi}$ for $1+\widehat{B}=f>0$, $\left(k \sim B i^{1 / 4}\right)$.

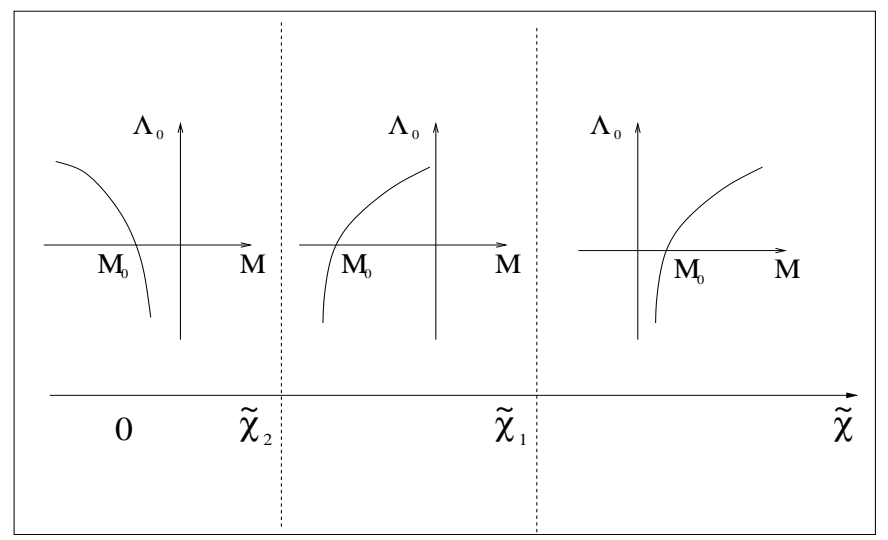

Figure 2: The dependence of the rescaled growth rate on the Marangoni number in the vicinity of the critical point $M_{0}=48 m_{0}$ in the different regions of $\tilde{\chi}$ for $1+\widehat{B}=f<0$, $\left(k \sim B i^{1 / 4}\right)$.

Using Eqs.(3.31), (3.32) we obtain the following relationship between the constants $a_{1}$ 
and $a_{2}$

$$
a_{2}=-\chi\left(1+L^{-1}\right) a_{1} .
$$

Without loss of generality we choose $a_{1}=1$, so that $a_{2}=-\chi\left(1+L^{-1}\right)$.

The solution of Eqs.(3.29) read

$$
\begin{gathered}
T_{2}=b_{1}+\frac{-4 K^{2}[2 z(5 z-12)+15] r_{0}[(L+1) \chi \varphi+L] z^{4}+9 K^{2} L\left[2(3 z-5) z^{2}+5\right] z^{2}}{90 L} \\
C_{2}=b_{2}+\frac{2 K^{2}(L+1) z^{4}[2 z(5 z-12)+15] \chi r_{0}[L+(L+1) \chi \varphi]}{45 L^{2}}- \\
\frac{K^{2}(L+1) z^{2}\left[2(3 z-5) z^{2}+5\right] \chi}{10 L} \\
\Psi_{2}=\frac{1}{45360 L(\chi L+L+\chi)}\left\{i K ( z - 1 ) z ^ { 2 } \left(6 0 4 8 0 L b _ { 1 } \left(r_{0}(2 L(5 z-3)+5(L+1)(2 z-3) \chi+\right.\right.\right. \\
9(L+1) \chi \varphi)-9 L)+\frac{1}{L}\left\{60480 L^{2} b_{2}\left(9 L+r_{0}(-(5 L(2 z-3)+2(L+1)(5 z-3) \chi) \varphi-9 L)\right)-\right. \\
(\chi L+L+\chi)\left(18144 K^{2} z(3 z-2) L^{2}+11340(\chi L+L+\chi) M_{2} L+\right. \\
64(10 z(z(z(z(z(2(z-3) z+3)+3)+3)+3)+3)+153) r_{0}^{2}(-(L+1) \chi \varphi-L) K^{2}+ \\
\left.\left.\left.\left.\left.945 L(2 z-3) R_{2}\right)\right)\right\}\right)\right\}
\end{gathered}
$$

At fourth order for the heat and mass diffusion equations are, respectively,

$$
\begin{gathered}
T_{4}^{\prime \prime}-K^{2} T_{2}=\omega_{0} P T_{2}+\omega_{2} P T_{0}+i K \Psi_{2} \\
C_{4}^{\prime \prime}-K^{2} C_{2}+\chi\left(T_{4}^{\prime \prime}-K^{2} T_{2}\right)=\omega_{0} P L^{-1} C_{2}+\omega_{2} P L^{-1} C_{0}-i K L^{-1} \chi \Psi_{2},
\end{gathered}
$$

with boundary conditions

$$
\begin{gathered}
z=0: \quad T_{4}^{\prime}=C_{4}^{\prime}=0 \\
z=1: \quad T_{4}^{\prime}+\beta T_{0}=0, \quad C_{4}^{\prime}-\chi \beta T_{0}=0 .
\end{gathered}
$$

The solvability conditions at fourth order are obtained by integration of Eqs.(3.42) over $0 \leq z \leq 1$,

$$
\begin{gathered}
-\beta T_{0}-K^{2}<T_{2}>=\omega_{2} P a_{1}+i K<\Psi_{2}> \\
\chi \beta T_{0}-K^{2}<C_{2}>+\chi\left[-\beta T_{0}-K^{2}<T_{2}>\right]=\omega_{2} P L^{-1} a_{2}-i K L^{-1} \chi<\Psi_{2}>,
\end{gathered}
$$

and substituting Eqs.(3.41) into Eqs.(3.44) yields

$$
\mathbf{A}_{\mathbf{2}}\left(\begin{array}{l}
b_{1} \\
b_{2}
\end{array}\right)=\left(\begin{array}{l}
q_{1} \\
q_{2}
\end{array}\right)
$$

with

$$
\mathbf{A}_{2}=\left(\begin{array}{cc}
\frac{(1+L)\left(-1+r_{0}(1-\varphi)\right) \chi}{L+\chi+L \chi} & \frac{L\left(-1+r_{0}(1-\varphi)\right)}{L+\chi+L \chi} \\
-\frac{(1+L) \chi\left(L+(1+L) \chi r_{0}(1-\varphi)\right)}{L(L+\chi+L \chi)} & -\frac{L+(1+L) \chi r_{0}(1-\varphi)}{L+\chi+L \chi}
\end{array}\right)
$$


where

$$
\begin{array}{r}
q_{1}=\frac{-1}{665280 L^{2}}\left\{-44352 L^{2}\left(K^{4}+15 \beta\right)+\right. \\
K^{2}\left(13860 L(L+\chi+L \chi) M_{2}+(-L-(1+L) \chi \varphi)\left(26752 K^{2} L r_{0}-2079 L R_{2}+\right.\right. \\
\left.\left.\left.15424 K^{2} r_{0}^{2}(-L-(1+L) \chi \varphi)\right)\right)-665280 L^{2} P \omega_{2}\right\} \\
q_{2}=\frac{1}{665280 L^{3}}\left\{\chi \left(13860 K^{2} L(1+L)(L+\chi+L \chi) M_{2}+\right.\right. \\
15424 K^{4}(1+L) r_{0}^{2}(L+(1+L) \chi \varphi)^{2}+ \\
26752 K^{4} L(1+L) r_{0}(-L-(1+L) \chi \varphi)-693 L\left(64 L\left(K^{4}(1+L)+15 L \beta\right)+\right. \\
\left.\left.\left.3 K^{2}(1+L) R_{2}(-L-(1+L) \chi \varphi)+960\left(1+L+L^{2}\right) P \omega_{2}\right)\right)\right\} .
\end{array}
$$

As operator $\mathbf{A}_{\mathbf{2}}$ is singular, according to the Fredholm theorem a solution exists if and only if vector $\mathbf{q}=\left(\begin{array}{l}q_{1} \\ q_{2}\end{array}\right)$ is orthogonal to the eigenvector $\mathbf{s}$ corresponding to a zero eigenvalue of the adjoint operator $\mathbf{A}_{\mathbf{2}}^{\dagger}$, i.e., $\mathbf{A}_{\mathbf{2}}^{\dagger} \mathbf{s}=0$,

$$
\mathbf{s}=\left(\begin{array}{c}
-\frac{L+(1+L) \chi r_{0}(1-\varphi)}{L\left(-1+r_{0}(1-\varphi)\right)} \\
-1
\end{array}\right)
$$

It follows from the orthogonality

$$
\mathbf{q} \cdot \mathbf{s}=0
$$

that

$$
\begin{gathered}
\omega_{2}=-\frac{L \beta\left[L(1+\chi)+\chi r_{0}(1-\varphi)\right]}{P\left[\chi+L(L+\chi+L \chi)-\chi r_{0}(1-\varphi)\right]}+ \\
\frac{K^{2}(L+\chi+L \chi)\left[20(L+\chi+L \chi) M_{2}+3 R_{2}(L+(1+L) \chi \varphi)\right]}{960 P\left[\chi+L(L+\chi+L \chi)-\chi r_{0}(1-\varphi)\right]}+ \\
\frac{K^{4}(L+\chi+L \chi)\left\{-693 L^{2}+r_{0}[-L-(1+L) \chi \varphi]\left[418 L+241 r_{0}(-L-(1+L) \chi \varphi)\right]\right\}}{10395 L P\left[\chi+L(L+\chi+L \chi)-\chi r_{0}(1-\varphi)\right]} .
\end{gathered}
$$

Equation (3.45) reduces in the case of no buyoancy, $r_{0}=R_{2}=0$, to the expression found for the pure Marangoni instability given in Eq.(23) in Ref. [15]. In the limit $K^{2} \gg L, r_{0} \neq 0$, the leading order expression for the growth rate $\omega_{2}$ reads

$$
\omega_{2}=-\frac{241 K^{4} \varphi^{2} \chi^{2} r_{0}{ }^{2}}{10395 L P\left[-1+(1+\varphi) r_{0}\right]},
$$

therefore, its value is negative and instability is long-wave when

$$
-1+(1+\varphi) r_{0}>0
$$


In the limit $K^{2}=O(L) \ll 1$, assuming $K^{2}=\delta L, \delta=O(1)$ in the leading order of approximation for the growth rate of disturbances we obtain

$$
\omega_{2}=\frac{L\left\{665280 \beta(1+\varphi) r_{0}+\delta \chi\left[-13860 M_{2}+\varphi\left(-15424 \delta \varphi \chi r_{0}^{2}+2079 R_{2}\right)\right]\right\}}{665280 P\left[-1+(1+\varphi) r_{0}\right]}
$$

Furthermore, consider the limit of both asymptotically small Lewis and Rayleigh numbers $L \sim r_{0} \ll 1$. In this case we obtain in the leading order of approximation with respect to small $L$

$$
\omega_{2}=\frac{K^{2} \chi\left(20 M_{2}+3 \varphi R_{2}\right)}{960 P}
$$

Thus, the monotonic instability is long-wave when the expression (3.48) has a positive sign, i.e., the Soret number $\chi$ and $\left(20 M_{2}+3 \varphi R_{2}\right)$ are of the same sign. It should be noted that assuming in addition $R_{2} \rightarrow 0$, the expression (3.48) completely matches the results from Ref. [15] (see there the growth rate Eq. (23) in the limit of small $L$ ).

\subsubsection{Oscillatory instability}

The oscillatory instability threshold is determined by the relation $\Lambda_{0}=i \Omega_{0}$ where $\Omega_{0}$ is real. In this case, the dispersion relation (3.33) then assumes the form

$$
\begin{gathered}
L-(L+\chi+L \chi) m_{0}+(-L-(1+L) \varphi \chi) r_{0}+ \\
i P\left(1+L-(1+\chi) m_{0}+(-1-\varphi \chi) r_{0}\right) \Omega_{0}-P^{2} \Omega_{0}^{2}=0,
\end{gathered}
$$

and by separating it into the real and imaginary parts we find the threshold for oscillatory instability

$$
m_{0}=\frac{1+L}{1+\widehat{B}+\tilde{\chi}}
$$

and the squared critical frequency

$$
\Omega_{0}^{2}=-\frac{\tilde{\chi}+L[\tilde{\chi}+L(1+\widehat{B}+\tilde{\chi})]}{P^{2}(1+\widehat{B}+\tilde{\chi})} .
$$

The expression (3.50) can be recast in the form

$$
\frac{m_{0}}{m_{*}}+\frac{r_{0}}{r_{*}}=1
$$

where $m_{*}=\frac{1+L}{1+\chi}, r_{*}=\frac{1+L}{1+\varphi \chi}$. Therefore, the regions of instability are determined by the signs of the expressions $1+\chi$ and $1+\varphi \chi$ as shown in Fig.3. 
A. Podolny et al.
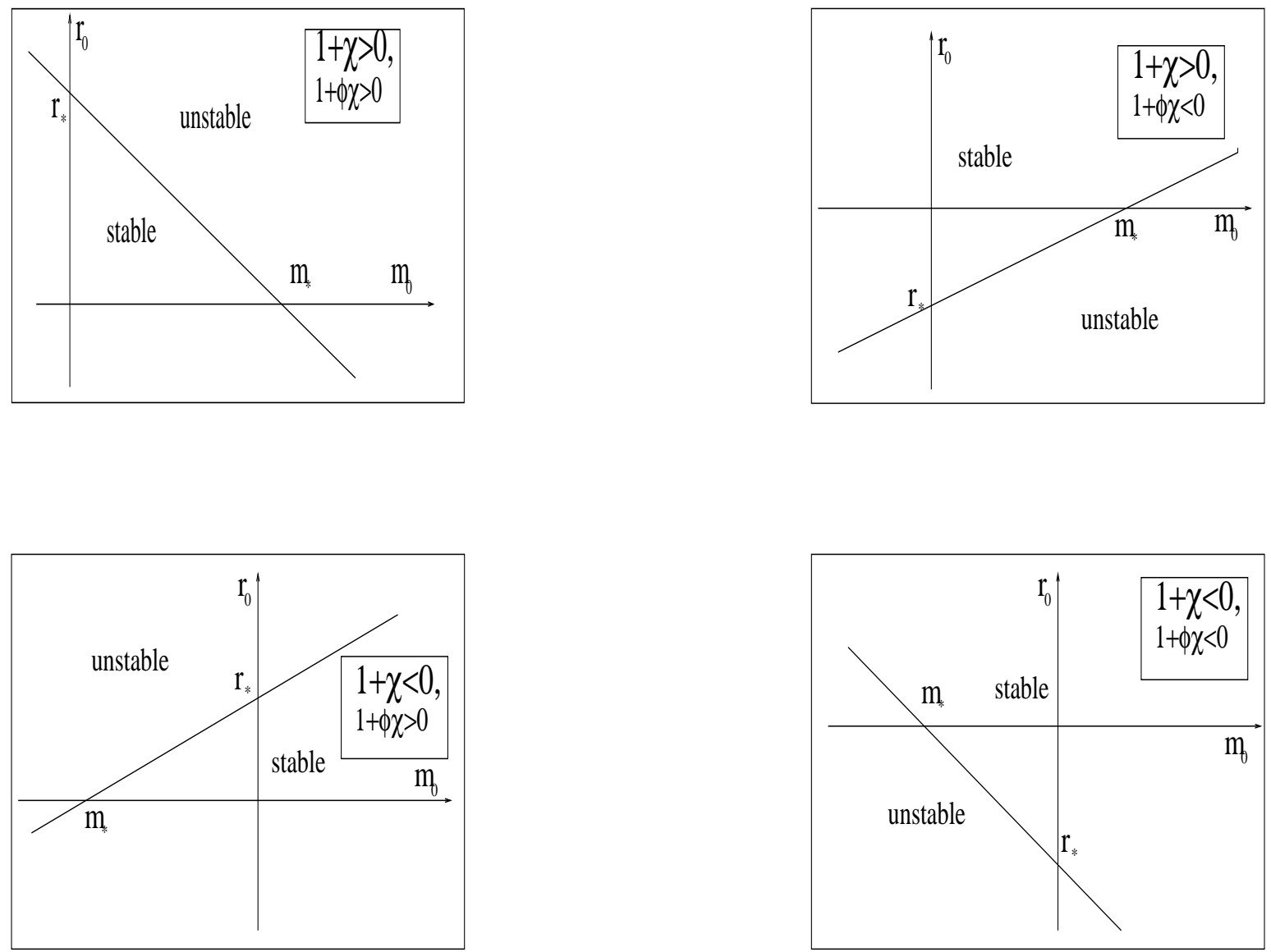

Figure 3: Variation of the oscillatory instability threshold given by Eq.(3.52) with Soret number $\chi$ and the separation ratio $\varphi$ in the long-wave region $k \sim B i^{1 / 4}$.

In the case of oscillatory instability, the solvability condition at fourth order determines the second-order correction $\omega_{2}$ to the complex growth rate. We present here only the expression for the real part of the coefficient $\omega_{2}$ which determines the growth rate of the oscillatory 
instability

$$
\begin{gathered}
R e\left[\omega_{2}\right]=\frac{-\beta}{2 P}+\frac{K^{2}\left(20(1+\chi) M_{2}+3(\varphi \chi+1) R_{2}\right)}{1920 P}+ \\
\left\{K ^ { 4 } \left(6 9 3 ( 1 + L ) \left(2 P \chi+L^{2}(-3+2 P)(1+\chi)-\right.\right.\right. \\
L(3 \chi+2 P(2+\chi)))+11\left(L^{2}(-9+170 P)(1+\chi)(-1-\varphi \chi)+\right. \\
2 P \chi(-111-\varphi(-26+85 \chi))+L(9 \chi(21-20 \varphi+\varphi \chi)+ \\
2 P(-76+\chi(-187-50 \varphi-161 \varphi \chi)))) r_{0}+ \\
\left.\left.4 P(-1-\varphi \chi)(241 L(1+\chi)(-1-\varphi \chi)+\chi(-264+23 \varphi-241 \varphi \chi)) r_{0}^{2}\right)\right\} / \\
{\left[83160 L P^{2}(1+\chi)\right] .}
\end{gathered}
$$

In the limit of small Lewis numbers with $r_{0} \neq 0$, the leading order of $R e\left[\omega_{2}\right]$ reads

$$
\operatorname{Re}\left[\omega_{2}\right]=\frac{K^{4} \chi\left[693-11[\varphi(85 \chi-26)+111] r_{0}+2(\varphi \chi+1)[\varphi(241 \chi-23)+264] r_{0}^{2}\right]}{41580 L P(1+\chi)}
$$

The existence of the long-wave instability is again determined by the sign of the expression (3.54). Re $\left[\omega_{2}\right]$ changes its sign when either $\chi=0, \chi=-1$ or

$$
r_{0}^{(1),(2)}=\frac{1386}{1221+\varphi(286-935 \chi) \pm \sqrt{11 \Delta}}
$$

where $\Delta=2475+150 \varphi(346+313 \chi)+\varphi^{2}(7436-\chi(37028+41989 \chi))$. The value of $\Delta$ changes its sign at

$$
\varphi_{1,2}=-\frac{165}{1730+1565 \chi \pm 456 \sqrt{14}(1+\chi)}
$$

$\varphi_{1}$ and $\varphi_{2}$ change their sign through infinity at $\chi=-1.05044$ and $\chi=0.16859$, respectively. Our analysis shows that when $\chi<-1.05044$ the real part of the growth rate is negative and the long-wave oscillatory instability sets in the domain $\varphi_{2}<\varphi<\varphi_{1}$ for $r_{0}^{(1)}<r_{0}<r_{0}^{(2)}$; when $-1.05044<\chi<-1$ the domain of long-wave instability sets in outside the interval $\left(\varphi_{1}, \varphi_{2}\right)$ for $r_{0}^{(1)}<r_{0}<r_{0}^{(2)}$; in the region $-1<\chi<0$, the real part of the growth rate is negative outside the interval $\left(\varphi_{2}, \varphi_{1}\right)$ for $r_{0}<r_{0}^{(1)}$ and for $r_{0}>r_{0}^{(2)}$ and inside the interval $\left(\varphi_{2}, \varphi_{1}\right)$ for all values of $r_{0}$; in the region $0<\chi<0.16859$, the long-wave oscillatory instability sets in outside the interval $\left(\varphi_{2}, \varphi_{1}\right)$ for $r_{0}^{(1)}<r_{0}<r_{0}^{(2)}$. Finally, in the domain $\chi>0.16859$, the long-wave oscillatory instability sets in when $\varphi_{1}<\varphi<\varphi_{2}$ for $r_{0}<r_{0}^{(1)}$ and for $r_{0}>r_{0}^{(2)}$.

A summary of the instability types in the limit $k \sim B i^{1 / 4}$ depending on the parameters of the problem, such as the dynamic Bond number $\widehat{B}, \chi$ and $\varphi$ is given in Figs. 4 and 5. In the case of $f=1+\widehat{B}>0$, the instability is monotonic for $M>0$ with $\tilde{\chi}>\tilde{\chi}_{2}$, oscillatory for $M>0$ with $-f<\tilde{\chi}<\tilde{\chi}_{2}$, and monotonic for $M<0$ with $\tilde{\chi}<\tilde{\chi}_{1}$. In the case of $f=1+\widehat{B}<0$, the instability is monotonic for $M>0$ with $\widetilde{\chi}>\widetilde{\chi}_{1}$, for $M<0$ 


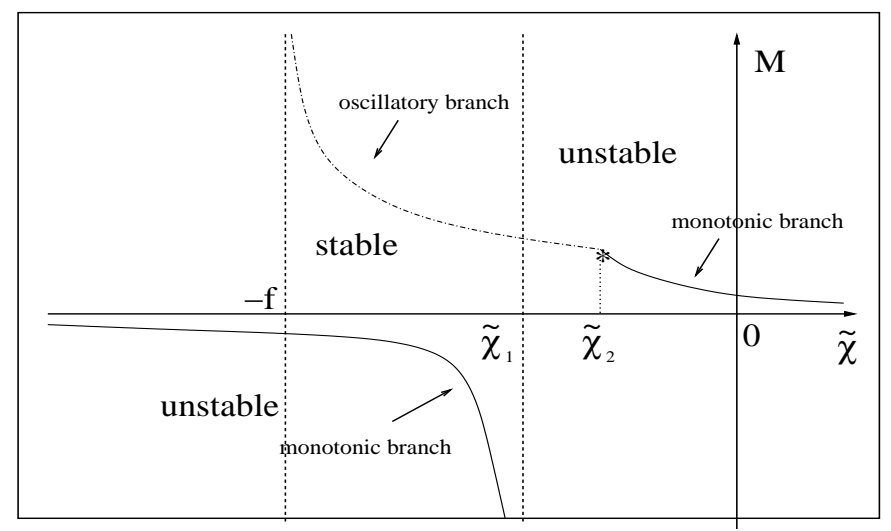

Figure 4: Neutral curves for both long-wave monotonic and oscillatory instabilities for $1+\widehat{B}=$ $f>0,\left(k \sim B i^{1 / 4}\right)$.

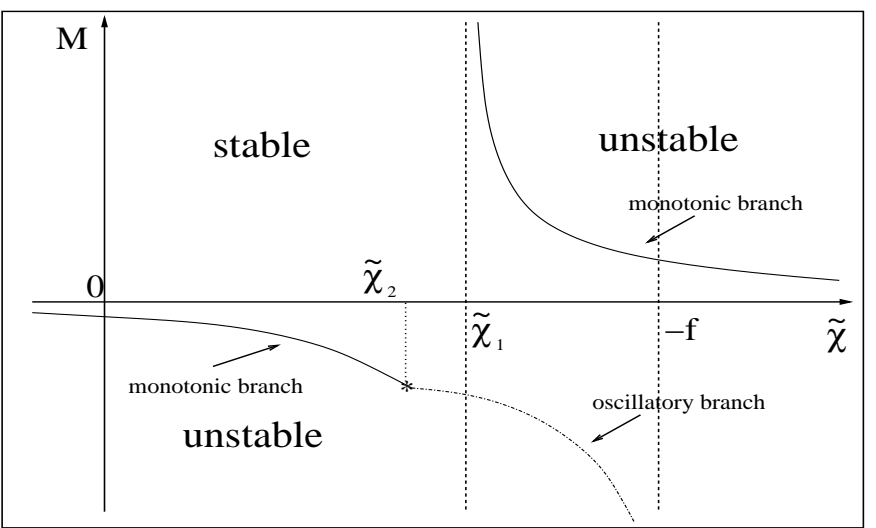

Figure 5: Neutral curves for both long-wave monotonic and oscillatory instabilities for $1+\widehat{B}=$ $f<0,\left(k \sim B i^{1 / 4}\right)$.

with $\tilde{\chi}<\tilde{\chi}_{2}$. and oscillatory for $M<0$ with $\tilde{\chi}>\tilde{\chi}_{2}$. An apparent symmetry of the neutral curves with respect to the horizontal axis follows from the symmetry of the critical values of the Marangoni number given by Eqs. (3.36), (3.50), i.e., $m_{0}(f, \tilde{\chi})=-m_{0}(-f,-\tilde{\chi})$, for both monotonic and oscillatory instabilities. The monotonic instability curve breaks up at the point $\tilde{\chi}_{1}$. The oscillatory instability curve breaks up at the point $\tilde{\chi}=-f$. Both neutral curves merge at the point $\tilde{\chi}_{2}$. When $f=1+\widehat{B}$ is positive $-f<\widetilde{\chi}_{1}<\tilde{\chi}_{2}$. Thus the instability is monotonic with $M<0$ for $\tilde{\chi}<\tilde{\chi}_{1}$, oscillatory instability with $M>0$ appears for $-f<\tilde{\chi}<\widetilde{\chi}_{2}$ and monotonic one with positive $M$ takes place for $\tilde{\chi}>\tilde{\chi}_{2}$. When $f=1+\widehat{B}<0 \tilde{\chi}_{2}<\tilde{\chi}_{1}<-f$. Thus the instability is monotonic with $M<0$ for $\tilde{\chi}>\tilde{\chi}_{1}$, oscillatory instability with $M<0$ appears for $\tilde{\chi}_{2}<\tilde{\chi}<-f$ and monotonic instability with negative $M$ takes place for $\tilde{\chi}<\tilde{\chi}_{2}$. 


\subsection{The case of $k=O\left(B i^{1 / 2}\right)$}

\subsubsection{Monotonic instability}

To complete the linear stability analysis, we now consider the limit $k \sim O\left(B i^{1 / 2}\right)(k=\widetilde{\varepsilon} q$, $B i=\widetilde{\varepsilon}^{2} \widetilde{\beta}$ with $\widetilde{\varepsilon} \ll 1$.

As a result, at zero order in $\widetilde{\varepsilon}$ we obtain Eqs. (3.27) with the solution given by Eq.(3.28) where the transformation $\varepsilon \rightarrow \widetilde{\varepsilon}, \beta \rightarrow \widetilde{\beta}, K \rightarrow q$ has to be made.

At second order the equations and boundary conditions upon substitution $\omega=0$ read

$$
\begin{gathered}
\Psi_{2}^{\prime \prime \prime \prime}-2 q^{2} \Psi_{0}^{\prime \prime}-i q R_{0}\left(T_{2}-\varphi C_{2}\right)-i q R_{2}\left(T_{0}-\varphi C_{0}\right)=0, \\
T_{2}^{\prime \prime}-q^{2} T_{0}=i q \Psi_{0}, \\
C_{2}^{\prime \prime}-q^{2} C_{0}+\chi\left(T_{2}^{\prime \prime}-q^{2} T_{0}\right)=-i q \chi L^{-1} \Psi_{0}, \\
z=0: \quad \Psi_{2}=\Psi_{2}^{\prime}=0, \quad T_{2}^{\prime}=C_{2}^{\prime}=0 \\
z=1: \quad \Psi_{2}=0, \quad T_{2}^{\prime}+\widetilde{\beta} T_{0}=0, \quad C_{2}^{\prime}-\chi \widetilde{\beta} T_{0}=0, \\
\Psi_{2}^{\prime \prime}=i q M_{0}\left[C_{2}-T_{2}\right]+i q M_{2}\left[C_{0}-T_{0}\right] .
\end{gathered}
$$

Integrating of the second and third equations in Eqs. (3.57) with respect to $z$ over $0 \leq z \leq 1$ yields the solvability conditions at second order

$$
\begin{gathered}
{\left[-\widetilde{\beta}+q^{2}\left(-1+m_{0}+r_{0}\right)\right] T_{0}-q^{2}\left[m_{0}-r_{0} \varphi\right] C_{0}=0,} \\
T_{0}\left\{-\widetilde{\beta} \chi+q^{2}\left(1+L^{-1}\right) \chi\left(m_{0}+r_{0}\right)\right\}+q^{2} C_{0}\left\{1-\left(1+L^{-1}\right) \chi\left(r_{0} \varphi+m_{0}\right)\right\}=0 .
\end{gathered}
$$

Thus, in the case of the monotonic instability, the neutral curve is determined at leading order by the expression

$$
m_{0}=\frac{L\left(q^{2}+\widetilde{\beta}\right)}{\widetilde{\beta} \chi+q^{2}[\tilde{\chi}+L(1+\widetilde{\chi}+\widehat{B})]} .
$$

The derivative $\frac{d m_{0}}{d q^{2}}$ is positive when $\tilde{\chi}<-(1+\widehat{B})$, and negative when $\tilde{\chi}>-(1+\widehat{B})$.

The relationship between the temperature and concentration disturbances at the leading order in terms of the original variables $k$ and $B i$ is found from the solution in the form

$$
T_{0}=-\frac{k^{2} L}{\chi\left[B i+k^{2}(1+L)\right]} C_{0} .
$$

Equation (3.60) provides an explanation of the strong dependence of the critical Marangoni number on the wavenumber $k$ in the domain $k=O\left(B i^{1 / 2}\right)$. The contribution of the thermocapillary effect to the tangential stress balance is proportional to $k^{2} / B i$ in this domain. In 
the limit $k^{2} \ll B i$ (or $q^{2} \ll 1$ ), the solutocapillary effect is the dominant factor triggering the instability. For $q \rightarrow 0$, we obtain

$$
M_{0}(0)=\frac{48 L}{\chi}
$$

Note that this result can be rewritten in terms of the solutal Marangoni number $M_{s} \equiv$ $M \chi L^{-1}$ as

$$
M_{s 0}(0)=48
$$

which is the traditional critical value for the long-wave instability of the pure solutocapillary convection with impermeable boundaries [10].

It should be noted that in the limit $q \rightarrow \infty\left(k \sim B i^{1 / 2} \ll B i^{1 / 4}\right)$, we reproduce exactly the result for the threshold of monotonic instability, as obtained in the previous section for $k=O\left(B i^{1 / 4}\right)$.

\subsubsection{Oscillatory instability}

The derivative of the rescaled growth rate $\Lambda_{0}=\omega_{0} / q^{2}$ with respect to the Marangoni number at the point $M=M_{0}$ is given by the expression

$$
\frac{d \Lambda_{0}}{d m_{0}}=\frac{\left[\widetilde{\beta} \tilde{\chi}+q^{2}(\tilde{\chi}+L(f+\widetilde{\chi}))\right]^{2}}{P\left[q^{2}(2+L) \widetilde{\beta} \widetilde{\chi}+\widetilde{\beta}^{2} \tilde{\chi}+q^{4}[\tilde{\chi}+L(\tilde{\chi}+L(f+\widetilde{\chi}))]\right.},
$$

where $f=1+\widehat{B}$.

In the case considered here

$$
Q=m_{0} \frac{d \Lambda_{0}}{d m_{0}}=\frac{L\left(q^{2}+\widetilde{\beta}\right)\left[\widetilde{\beta} \widetilde{\chi}+q^{2}(\tilde{\chi}+L(f+\tilde{\chi}))\right]}{P\left[q^{2}(2+L) \widetilde{\beta} \widetilde{\chi}+\widetilde{\beta}^{2} \widetilde{\chi}+q^{4}(\widetilde{\chi}+L(\widetilde{\chi}+L(f+\widetilde{\chi})))\right]} .
$$

We find that in the limit $q \rightarrow 0, Q=\frac{1}{S}>0$, therefore the oscillatory instability is irrelevant in this limit.

We now investigate the emergence of oscillatory instability in various domains of the parameters $f$ and $\widetilde{\chi}$ and first consider the case of $f=0$. In this case, $\Omega_{0}^{2}$ given by

$$
\Omega_{0}^{2}=-\frac{f q^{4} L^{2}+\left[q^{4}\left(1+L+L^{2}\right)+q^{2}(2+L) \widetilde{\beta}+\widetilde{\beta}^{2}\right] \tilde{\chi}}{q^{4} P^{2}(f+\widetilde{\chi})}
$$

is always negative and oscillatory instability does not emerge. Thus, for positive (negative) values of $\tilde{\chi}$, the monotonic instability emerges in positive (negative) domains of the Marangoni number with $|M|_{\min }=\left|M_{0}(0)\right|$, see Fig.6a. 
The next case is that of $f>0$. The numerator of $Q$ changes its sign in the domain $\tilde{\chi}_{1}<\tilde{\chi}<0$ at the point $q_{1}=\sqrt{-\frac{\tilde{\beta} \tilde{\chi}}{\tilde{\chi}+L(f+\tilde{\chi})}}$, where the sign of $m_{0}(q)$ changes as well. The following conclusions can be thus made: (i) $\tilde{\chi} \geq 0$, then $M_{0}(q)>0$ for all $q$; (ii) $\tilde{\chi}_{1}<\tilde{\chi}<0$, then $M_{0}(q)<0$ for $q<q_{1}$ and $M_{0}(q)>0$ for $q>q_{1}$; (iii) $\tilde{\chi} \leq \tilde{\chi}_{1}$, then $M_{0}(q)<0$ for all $q$.

The denominator of $Q$ changes its sign at the wavenumber

$$
q_{2}=\sqrt{\frac{\widetilde{\beta}\left[(-2-L) \tilde{\chi}+\sqrt{-\left(L^{2} \widetilde{\chi}(4 f+3 \tilde{\chi})\right)}\right]}{2[\widetilde{\chi}+L(\widetilde{\chi}+L(f+\widetilde{\chi}))]}},
$$

in the domain $\tilde{\chi}_{2}<\tilde{\chi}<0$. Note that in the considered case of $f>0, \widetilde{\chi}_{1}<\widetilde{\chi}_{2}<0$. It should be also noted that in the domain of existence $\tilde{\chi}_{2}<\tilde{\chi}<0$, the relationship $q_{1}<q_{2}$ is valid.

The value $\tilde{\chi}_{2}$ where the numerator of the squared frequency $\Omega_{0}^{2}$ given by Eq.(3.65) changes sign. The following properties of the expression given by Eq.(3.65) are important to be noted. (i) $\tilde{\chi}>0, \Omega_{0}^{2}<0$ for all $q$; (ii) $\tilde{\chi}_{2}<\tilde{\chi}<0, \Omega_{0}^{2}>0$ for $q<q_{2}$ and $\Omega_{0}^{2}<0$ for $q>q_{2}$; (iii) $-f<\tilde{\chi} \leq \tilde{\chi}_{2}, \Omega_{0}^{2}>0$ for all $q$; (iv) $\tilde{\chi}<-f, \Omega_{0}^{2}<0$ for all $q$. It should be noted that negative sign of the $\Omega_{0}^{2}$ means absence of the oscillatory instability.

Based on these properties we obtain the following results:

(a) If $\tilde{\chi} \geq 0$, the value of $Q$ given by Eq.(3.64) is always positive, and the instability is always monotonic with $M_{0}(q)$ given by Eq.(3.59) and the critical Marangoni number is $M_{\text {min }}=M_{0}(\infty)=\frac{48}{f+\left(1+L^{-1}\right) \tilde{\chi}}$, as shown in Fig.6b.

(b) In the domain $-f<\tilde{\chi} \leq \widetilde{\chi}_{1}, Q$ is positive and the monotonic instability sets in when $M_{0}(q)<0$ with $|M|_{\text {min }}=\left|M_{0}(0)\right|$. At the same time there is oscillatory instability for positive $M_{o s c}$, as shown in Fig.6c.

(c) If $\tilde{\chi}<-f$, there exists only monotonic instability for negative $M_{0}(q)$ with $|M|_{\text {min }}=$ $\left|M_{0}(\infty)\right|$, as shown in Fig.6d.

(d) In the domain $\tilde{\chi}_{2}<\tilde{\chi}<0$, the longwave instability is monotonic in two intervals of the wavenumbers: (i) $q<q_{1}$ with $M_{0}(q)<0$; (ii) $q>q_{2}$ with $M_{0}(q)>0$. In the domain $q_{1}<q<q_{2}, Q$ is negative and oscillatory instability sets in with the positive critical value of

$$
M_{o s c}=\frac{48\left((1+L) q^{2}+\widetilde{\beta}\right)}{q^{2}(f+\widetilde{\chi})} .
$$

in the range for $q<q_{2}$, as shown in Fig.6e.

(e) In the domain $\widetilde{\chi}_{1}<\tilde{\chi} \leq \widetilde{\chi}_{2}$, there exist two distinct intervals of wavenumbers, namely: (i) $q<q_{1}$ with $M_{0}(q)<0$ and $M_{o s c}(q)>0$ (ii) $q>q_{1}$ with $0<M_{o s c}(q)<M_{0}(q)$, as shown in Fig.6f.

Finally, the case of $f<0$ is considered. The numerator of $Q$ changes its sign in the domain $0<\tilde{\chi}<\tilde{\chi}_{1}$ at the point $q_{1}=\sqrt{-\frac{\tilde{\beta} \widetilde{\chi}}{\tilde{\chi}+L(f+\tilde{\chi})}}$, where the sign of $M_{0}(q)$ changes 
as well. The following properties of $M_{0}(q)$ are to be noted.

(i) $\tilde{\chi} \geq \tilde{\chi}_{1}$ then $M_{0}(q)>0$ for all $q$; (ii) $0<\tilde{\chi}<\widetilde{\chi}_{1}$ then $M_{0}(q)>0$ for $q<q_{1}$ and $M_{0}(q)<0$ for $q>q_{1}$; (iii) $\tilde{\chi} \leq 0$ then $M_{0}(q)<0$ for all $q$.

The denominator of $Q$ changes its sign at the wavenumber

$$
q_{2}=-\frac{\beta\left[(2+L) \tilde{\chi}+\sqrt{-\left(L^{2} \tilde{\chi}(4 f+3 \tilde{\chi})\right)}\right]}{2[\tilde{\chi}+L(\tilde{\chi}+L(f+\tilde{\chi}))]},
$$

in the domain $0<\tilde{\chi}<\widetilde{\chi}_{2}$. It is important to note that $0<\widetilde{\chi}_{2}<\widetilde{\chi}_{1}$ is satisfied in the current case of $f<0$. It is also noted that $q_{1}<q_{2}$ in the domain of existence $0<\tilde{\chi}<\tilde{\chi}_{2}$.

The numerator of the squared frequency $\Omega_{0}^{2}$ given by Eq.(3.65) changes its sign at $\tilde{\chi}=\tilde{\chi}_{2}$. The following properties are important for the forthcoming analysis. (i) If $\tilde{\chi}>-f$ then $\Omega_{0}^{2}<0$ for all $q$; (ii) If $\tilde{\chi}_{2} \leq \tilde{\chi}<-f$ then $\Omega_{0}^{2}>0$ for all $q$; (iii) If $0<\tilde{\chi}<\tilde{\chi}_{2}$ then $\Omega_{0}^{2}>0$ for $q<q_{2}$ and $\Omega_{0}^{2}<0$ for $q>q_{2}$; (iv) If $\tilde{\chi} \leq 0$ then $\Omega_{0}^{2}<0$ for all $q$.

Based on these properties we find the following results for the instability thresholds in various parameter domains:

(a) In the domain $\tilde{\chi} \leq 0$, the value of $Q$ given by Eq.(3.64) is always positive, and the instability always monotonic with $M_{0}(q)<0$ given by Eq.(3.59) with the critical value of the Marangoni number $|M|_{\min }=\left|M_{0}(\infty)\right|$, as shown in Fig.7a.

(b) In the domain $\tilde{\chi}_{1} \leq \widetilde{\chi}<-f, Q$ is positive and the monotonic instability sets in when $M_{0}(q)>0$ with $M_{\min }=M_{0}(0)$. At the same time there exists oscillatory instability for negative $M_{o s c}$, as shown in Fig.7b. One may notice a symmetry of the oscillatory neutral curves under transformation $M_{o s c}(f, \tilde{\chi})=-M_{o s c}(-f,-\tilde{\chi})$ following from Eq. (3.67).

(c) In the domain $\tilde{\chi}<-f$, there exists only monotonic instability for positive $M_{0}(q)$ with $M_{\min }=M_{0}(\infty)$, as shown in Fig.7c.

(d) In the domain $0<\tilde{\chi}<\widetilde{\chi}_{2}$, the longwave instability is monotonic in two intervals of wavenumbers, namely: (i) $q<q_{1}$ with $M_{0}(q)>0$; (ii) $q>q_{2}$ with $M_{0}(q)<0$. In the domain $q_{1}<q<q_{2}, Q$ is negative, and oscillatory instability sets in with negative value of critical Marangoni number $M_{\text {osc }}$ for $q<q_{2}$, as shown in Fig.7d.

(e) In the domain $\widetilde{\chi}_{2} \leq \tilde{\chi}<\widetilde{\chi}_{1}$, the two following subdomains are relevant, (i) $q<q_{1}$ with positive $Q$, then $M_{0}(q)>0$ and $M_{o s c}(q)<0$ (ii) $q>q_{1}$ with negative $Q$, then $0>$ $M_{\text {osc }}(q)>M_{0}(q)$, as shown in Fig.7e.

A summary of the instability types in the limit $k \sim B i^{1 / 2}$ depending on the system parameters such as the dynamic Bond number $\widetilde{B}, \chi$ and $\varphi$, is given in Figs. 6 and 7 . 

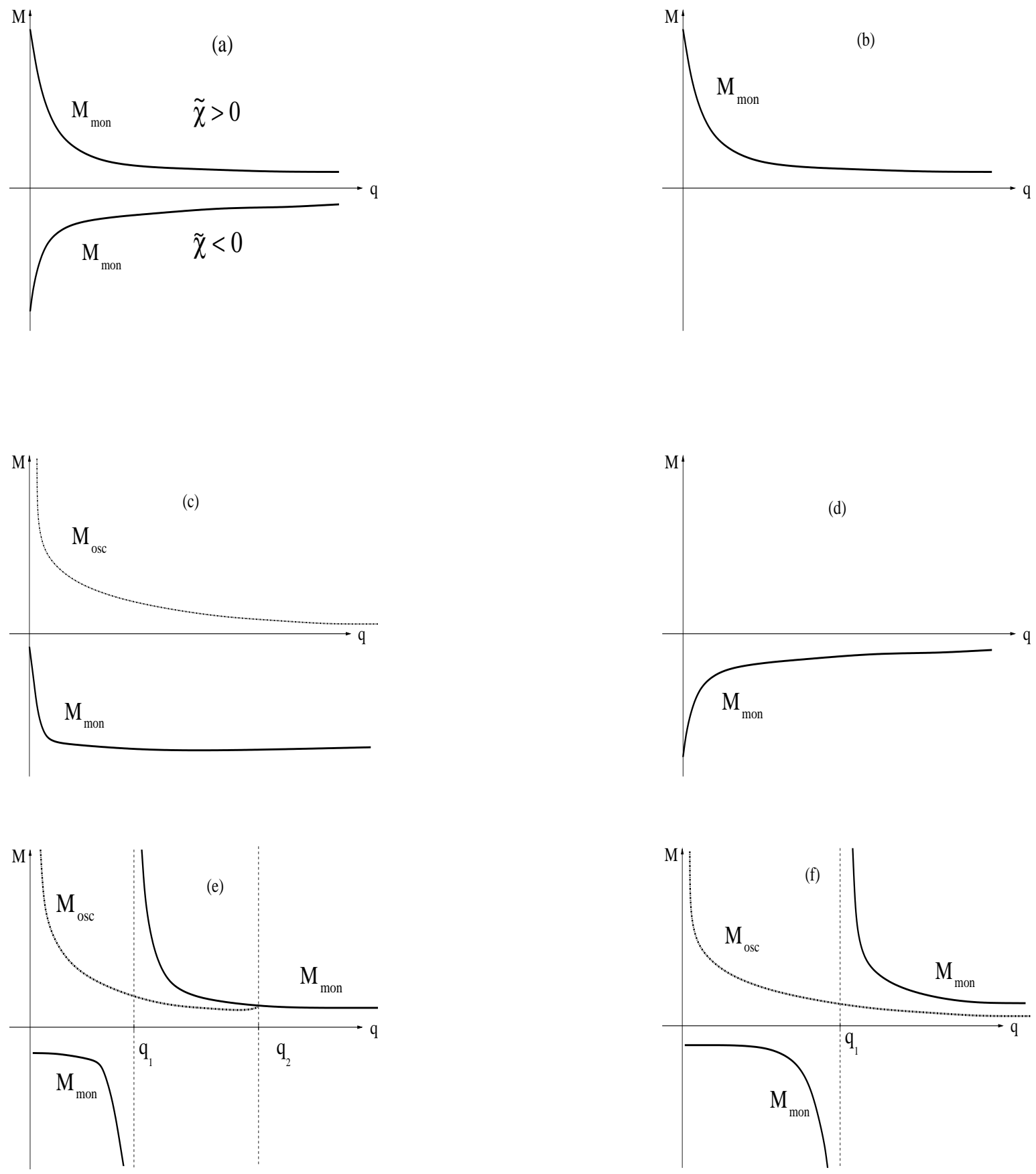

Figure 6: Neutral curves for both long-wave monotonic and oscillatory instabilities for $f \geq 0$, $\left(k \sim B i^{1 / 2}\right)$. 

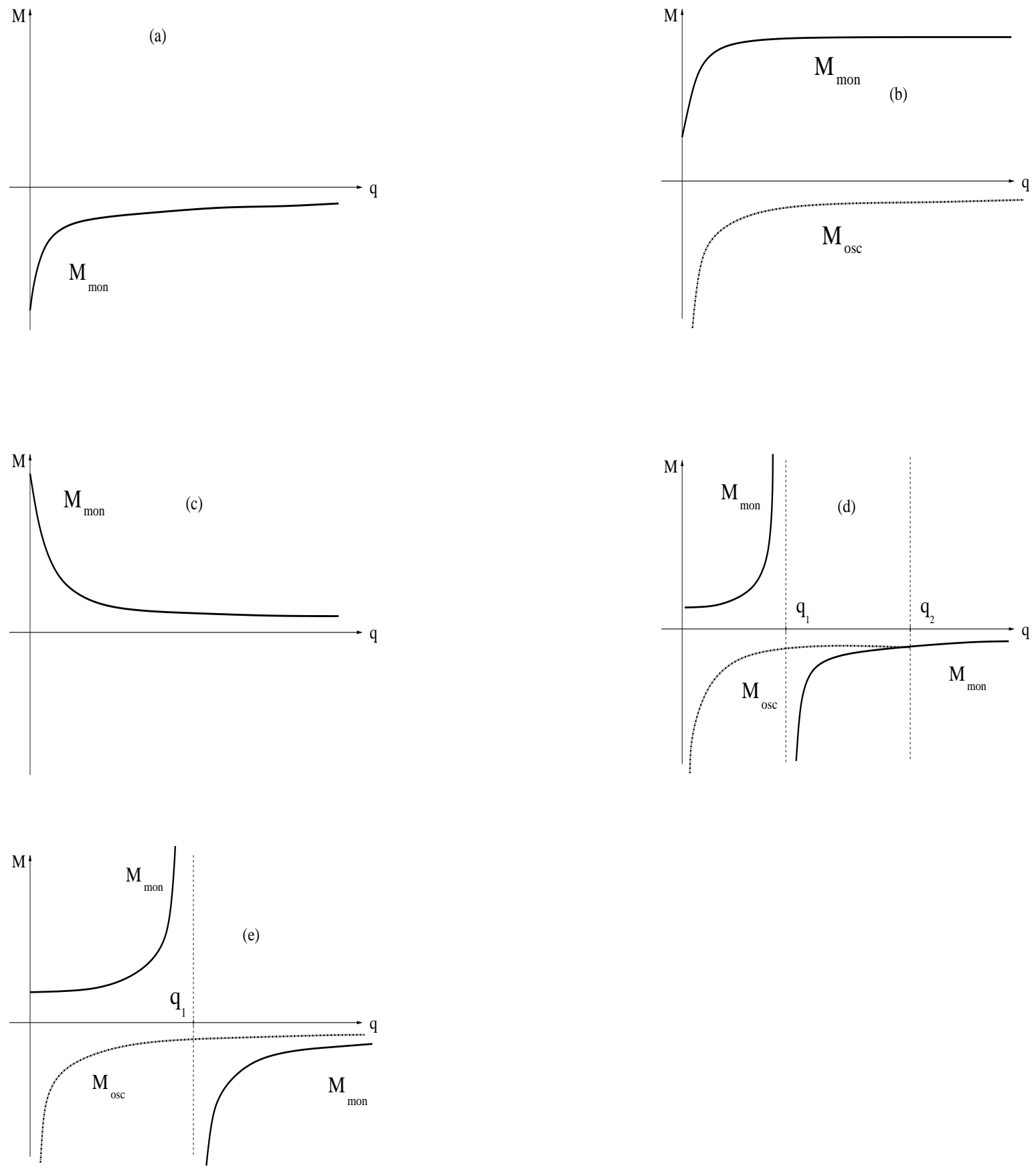

Figure 7: Neutral curves for both long-wave monotonic and oscillatory instabilities for $f<0$, $\left(k \sim B i^{1 / 2}\right)$. 


\section{Summary}

We have investigated the long-wave coupled Marangoni - Rayleigh instability of the quiescent state in a binary liquid layer with a nondeformable interface subjected to differential heating in the presence of the Soret effect. In the case of small Biot numbers $B i$, there exist two distinct long-wave regions, $k \sim B i^{1 / 4}$ and $k \sim B i^{1 / 2}$, where the topology of instability thresholds, both monotonic and oscillatory, is studied with respect to the Soret and dynamic Bond numbers.

A comparison of the behavior of the instability thresholds in both long-wave regions $k \sim B i^{1 / 4}$ and $k \sim B i^{1 / 2}$ with the corresponding results obtained in the limit of pure Marangoni instability [15], [18] shows qualitative differences in the case of negative $f$, as shown in Fig. 5 in the limit $k \sim B i^{1 / 4}$, and in Fig. 7 in the limit $k \sim B i^{1 / 2}$. In the case of positive $f=1+\widehat{B}$, the neutral curves shown in Fig. 4, are similar to those presented in Fig. 1 [15] in the limit $k \sim B i^{1 / 4}$ and the neutral curves shown in Fig.6 reproduce the results in the limit $k \sim B i^{1 / 2}$ presented in Sec.IV A, [18].

The linear stability analysis carried out in this paper reveals the diversity of instability types in the long-wave region, and a need in the development of the nonlinear theory to describe the evolution of the system beyond the linear stage is apparent.

\section{Acknowledgements}

A. A. N. acknowledges the support of Israel Ministry of Science (grant \#3-3570), Minerva Center for Nonlinear Physics of Complex Systems and Israel Science Foundation (grant \# 812/06). A. O. acknowledges the partial support of the Fund for Promotion of Research at the Technion and the Technion Research Vice-President Fund.

\section{References}

[1] J.L. Castillo, M.G. Velarde. Thermal diffusion and the Marangoni-Benard instability of a two-component fluid layer heated from below. Phys. Lett. A 66 (1978), 489-491.

[2] J.L. Castillo, M.G. Velarde. Microgravity and thermoconvective stability of a binary liquid layer open to the ambient gas. J. Nonequilib. Thermodyn., 5 (1980), 111-124.

[3] C.F. Chen, C.C. Chen. Effect of surface tension on the stability of a binary fluid layer under reduced gravity. Phys. Fluids, 6 (1994), 1482-1490.

[4] C.F. Chen, T.F. Su. Effects of surface tension on the onset of convection in a doublediffusive layer. Phys. Fluids A, 4 (1992), 2360-2367.

[5] P. Colinet, J.C. Legros, M.G. Velarde. Nonlinear Dynamics of Surface-Tension-Driven Instabilities. Wiley-VCH, Berlin, 2001. 
[6] M.C. Cross, P.C. Hohenberg. Pattern formation outside of equilibrium. Rev. Mod. Phys., 65 (1993), 851-1112, Sec. IXA.

[7] V.L. Gertsberg, G.I. Sivashinsky. Large cells in Rayleigh-Benard convection. Prog. Theor. Phys., 66 (1981), 1219-1229.

[8] S.R. de Groot, P. Mazur. Non-equilibrium thermodynamics. North-Holland, Amsterdam, 1962.

[9] S. Hollinger, M. Lücke, H.W. Miller. Model for convection in binary liquids. Phys. Rev. E, 57 (1998), 4250-4264.

[10] S.W. Joo. Marangoni instabilities in liquid mixtures with Soret effect. J. Fluid Mech. 293 (1995), 127-145.

[11] C.L. McTaggart. Convection driven by concentration- and temperature-dependent surface tension. J. Fluid Mech. 134 (1983), 301-310.

[12] A.A. Nepomnyashchy, M.G. Velarde, P. Colinet. Interfacial phenomena and convection. Chapman and Hall/CRC Press, London, 2001.

[13] D.A. Nield. The thermohaline Rayleigh-Jeffreys problem. J. Fluid Mech., 29 (1967), 545-558.

[14] D.A. Nield. Surface tension and buoyancy effects in cellular convection. J. Fluid Mech., 19 (1964), 341-352.

[15] A. Oron, A.A. Nepomnyashchy. Long-wavelength thermocapillary instability with the Soret effect. Phys. Rev. E, 69 (2994), 016313-1 - 016313-17.

[16] S. Ostrach. Fluid mechanics in crystal growth. J. Fluids Eng., 105 (1983), 5-20.

[17] J.R.A. Pearson. On convection cells induced by surface tension. J. Fluid Mech., 4 (1958), 489-500.

[18] A. Podolny, A. Oron, A.A. Nepomnyashchy. Long-wave Marangoni instability in a binary-liquid layer with deformable interface in the presence of Soret effect: Linear theory. Phys.Fluids, 17 (2006), 104104-1 - 104104-25.

[19] S. Shklyaev, A.A. Nepomnyashchy, A. Oron. Three-dimensional oscillatory long-wave Marangoni convection in a binary liquid layer with the Soret effect: Bifurcation analysis. Phys. Fluids, 19 (2007), 072105-1 - 072105-14.

[20] G.I. Sivashinsky. Large cells in nonlinear Marangoni convection. Phys. D, 4 (1982), pp. 227-235. 
[21] J.R.L. Skarda, D. Jacqmin, F.E. McCaughan. Exact and approximate solutions for the double diffusive Marangoni-Benard problem with cross-diffusive terms. J. Fluid Mech., 366 (1998), 109-133.

[22] S. Slavchev, G. Simeonov, S. Van Vaerenbergh, J.C. Legros. Marangoni instability of a layer of binary liquid in the presence of non-linear Soret effect. Int. J. Heat Mass Transfer, 42 (1999), 3007-3011.

[23] M. Takashima. Surface tension driven instability in a horizontal layer of binary liquid mixture in the presence of the Soret effect. J. Phys. Soc. Jpn, 47 (1979), 1321-1326.

[24] M. Takashima. Surface tension driven instability in a horizontal layer of binary liquid mixture in the presence of the Soret effect. II. Overstability. J. Phys. Soc. Jpn., 49 (1980), pp. 802-806.

[25] J.S. Turner. Double-diffusive phenomena. Ann. Rev. Fluid Mech., 6 (1974), 37-56.

[26] J.S. Turner. Multicomponent convection. Ann. Rev. Fluid Mech., 17 (1985), 11-44. 